\title{
A Study on Convergence, Divergence and Maintenance in OCD Patients
}

\author{
Daniela Stabile ${ }^{1}$, Francesca Scafuto ${ }^{2}$, Augusto Gnisci ${ }^{1}$, Alessandro Iavarone ${ }^{3}$ \\ ${ }^{1}$ Department of Psychology, Second University of Naples, Caserta, Italy \\ ${ }^{2}$ Department of Political Sciences, University of Naples, Federico II, Napoli, Italy \\ ${ }^{3}$ Department of Theories and Methods of Human and Social Sciences, University of Naples, Federico II, Napoli, Italy \\ Email: daniela_stabile@virgilio.it, francesca.scafuto@unina.it, augusto.gnisci@unina2.it, aleiavarone@libero.it
}

Received January 30, 2013; revised March 5, 2013; accepted March 15, 2013

Copyright (C) 2013 Daniela Stabile et al. This is an open access article distributed under the Creative Commons Attribution License, which permits unrestricted use, distribution, and reproduction in any medium, provided the original work is properly cited.

\begin{abstract}
The aim of this study was to verify if patients with Obsessive-Compulsive Disorder (OCD) adopted self-referential and non-adherent interactive modalities, during face-to-face conversation, to a higher extent as compared with subjects who did not have a diagnosis of OCD. For this purpose, four subjects with OCD and four age, sex and education matched Normal Controls (NC) underwent a semi-structured interview. The video-taped records have been evaluated and coded by means of the Initiative-Response Analysis system (I-R). The interview allowed us to obtain indexes of interactive strategies, namely, convergence, divergence and maintenance, which have been interpreted in the Communication Accommodation Theory (CAT) framework. Subjects with OCD, as compared with both NC and interviewers, mainly used Maintenance strategy, indicating a non-adherence to turns and an over-reliance on their own turns, thus neglecting the partners' contribution. This kind of strategy exclusively correlated with the scores of the Y-BOCS compulsion subscale. Results are consistent with the hypothesis that communication in subjects with OCD relies on particular strategies and support the view that communication is strongly correlated with "personological" variables. This hypothesis may be incorporated (not being incompatible with) in the CAT framework.
\end{abstract}

Keywords: CAT; Communication; Clinical Psychology; Personality Disorder; Initiative-Response Analysis; OCD; Y-BOCS; DSM IV-TR

\section{Introduction}

\subsection{The Accommodation Strategies}

The Communication Accommodation Theory [1] states that the communicative act may be viewed in terms of identity affirmation and relationship. The CAT offers an interdisciplinary model to understand processes of relation and identity in the interactive social context, thus emphasizing the relevance of linguistic behaviors in both face-to-face and group communication. The main hypothesis of CAT is that individuals use language to create a willing social distance between themselves and the others. Thus, language and language behaviors may be used to mark ethnical differences, social role, limits between or within the groups, as if to reinforce role- and norm-specific behaviors. Communicative strategies of convergence, divergence and maintenance [2] would empower individuals to adapt or outdistance their communicative acts to those of the conversation partner.

In communicative turns, when individuals move to- ward a convergent direction, communicative modalities of both partners become similar, assuming more homogeneous features; in the opposite case (divergent direction) differences between partners progressively increase. The consequence of this differentiation could generate a condition that could be defined, in theoretical terms, "schismogenesis" [3].

When maintenance strategies are prevalent, interactive turns are characterized by the relying on the communicative style of only one partner, in spite of the attempts of the interlocutor to shift on accommodation $[4,5]$. This strategy has been viewed as a particular form of divergence, being psychologically an equivalent of the last. However, all convergent strategies establish homogeneous patterns of communication, namely, while divergent strategies determine discordant ones, and maintenance strategies bring self-referred patterns $[5,6]$.

With respect to the raters of communicative interaction, three distinct types of accommodation are possible. The evaluation carried out by independent raters (i.e. not in- 
volved in the communication) is called "objective accommodation" whereas the self-judgment by the speaker is defined as "psychological accommodation"; finally, the term "perceptual/subjective accommodation" refers to the perceiving of the communicative state by the partner [2]. In the present study, the evaluation has been performed by means of the first type of accommodation, since assessment procedures were completed by two independent raters. This choice allowed us to evaluate the so-called objective communicative behavior through direct observations of linguistic interactions.

Data from literature have highlighted implicit factors involved in communicative interaction, namely, group, inter-personal and intra-personal factors. The group factor refers to the perception of their own group given by individuals. At this purpose, the term upward accommodation defines the adaptation of communicative strategies toward the group recognized as the more prestigious. Interpersonal factors are referred to dimensions of social desirability, in which norms, stereotypes and expectations converge, all of them contributing to give a picture to the interlocutor. Finally, intra-personal factors refer to the subjective perception of its own identity; the role of the last factors has been mainly considered in the study of perceptual/subjective accommodation.

Several recent studies have addressed the topics of the shifting of linguistic and paralinguistic codes, for example, accent and speed of talking [1] in communicative settings like job sites $[7,8]$, inter-generation or transcultural meetings [9-11].

The patient-physician communicative context has also been studied. Contributions from literature have confirmed the role of several variables like social status, and kind of symptoms referred by patients. Moreover, it has been pointed to the role played by some personality traits, like optimism vs pessimism, conservative vs progressive and agitated vs quiet [1].

The CAT is a setting-based theory, which takes into account proximal determinants of interactive behavior. These determinants incorporate the linguistic act formerly conceived by a partner, the cognitive mediations arising to processes of social categorization, and stereotypes toward the interlocutor [12]. On the other hand, the CAT does not rule out, from its framework, the influence of possible individual differences [5]. According to this hypothesis, our aim was to evaluate the role of psychopathological factors in a context different from the usual patient-physician interview. Indeed, the setting we adopted was an interview about general topics other than patients' disorders, carried on without any previous relationship or familiarity between the partners. The main assumption supporting this choice is that a set of predominant communicative strategies may be looked as a style of interaction, which can be influenced (or at least correlated) by behavioral dimensions of specific psychopathological disorders.

It should be of interest to look at accommodation strategies not only in terms of social-cognitive processes. In fact, along with some studies [2], the CAT could also provide a theoretical framework to explain some features of psychopathological and behavioral disorders.

\subsection{The Study: Objective and Hypothesis}

Objective of the present study was to evaluate the characteristics of interactive strategies in subjects with cognitive and behavioral symptoms of Obsessive Compulsive Disorder (OCD), as compared to those of matched Normal Controls (NC). All participants underwent a semistructured interview with a specially trained interviewer. The video-taped material has been evaluated by means of a codified system which allowed detecting the presence of interactive strategies of Convergence, Divergence and Maintenance. The main hypothesis was that, given the cognitive inflexibility, subjects with OCD could exhibit a parallel communicative rigidity, which could be expressed by reduced flexibility in shifting from an argument to another, in a focal non-adherence to adjacent turns, and tendency to self-reference, thus over-relying on maintenance strategy. This communicative pattern can be related to the pervasive features of the "obsessive thought", which is often described as scrupulous, doubting and hyper-attentive, with an increasing expression of these features with the severity of the disorder $[13,14]$. The specific objective of the study was to verify if, during interview, patients with OCD significantly over-relied on Maintenance strategy as compared with NC. As consequence, we hypothesized that the obliqueness index (according to the Initiative-Response Analysis, corresponding to the Maintenance of the CAT) would be higher in the OCD group, as if it should be different between interviewer and interviewed. We also hypothesized a positive correlation between obliqueness variable and severity of psychopathology, that is, the pervasiveness of obsessions and compulsions.

\section{Methods}

\subsection{Subjects}

Four patients with diagnosis of OCD according to DSM IV-TR criteria [23] have been involved into the present study. They were two men and two women, with age ranging from 34 to 44 years. They were recruited among outpatients referring to the Department of Mental Health of Caserta, Italy. All patients assumed psychotropic medication to treat symptoms of OCD and were involved in a psychotherapeutic program at the time of evaluation. The four patients belonging to the experimental group were matched with normal controls (NC). They were 
four subjects free from psychiatric disorders, comparable to OCD group for age, sex and education (years of schooling) [15]. All subjects gave their consent to the interview, but they should not deduce the real purpose of the study.

\subsection{Procedures}

All subjects underwent a semi-structured interview, lasting about $20 \mathrm{~min}$. Therefore, a pool of $160 \mathrm{~min}$ of taped material has been evaluated ( $80 \mathrm{~min}$ for experimental and 80 min for the control group).

The interview starts with questions aimed to obtain general information about the subject (i.e. age, marital condition, job, living quarter). Then follows interactive, open-format questions aimed to give interviewees the perception of a relatively informal interaction, to express his/her point of view. The subjects knew they had been involved in a study on personal opinions about general topics, like inflation, environmental protection, generation gap etc. All subjects completed the interview at their home.

The interviewer did not just formulate questions, but also participated with his statements as in an informal conversation.

Some of the questions, used in the interview, are reported as follows:

I: About the high cost of living, do you think that purchasing power of consumers is really diminished in recent years? Why?

I: Do you know the new law about forbidden smoking in public places, which punishes up to 2000 euro fine the owners of public places who do not respect the prohibition? Do you believe this action enough to prevent persons from smoking? Why?

I: Have you ever smoked in public places?

I: Would you tell me how did you feel when you have been asked to move outside the place to smoke?

Diagnosis: The Yale Brown Obsessive Compulsive Scale [17] has been used to assess symptoms of OCD and to grade the severity of the disorder. The Y-BOCS is divided into three sections. The first section (Y-BOCS$\mathrm{CC}$ ) evaluates Core Components of the OCD by means of ten items, half of them assessing obsessions and the remaining compulsions. The scores of each single item range from 0 to 4 , with 4 corresponding to the higher level of severity of the symptom. The dimensions taken into account to evaluate obsessions and compulsions are time spent, interference, distress, resistance and control. The second section, i.e., Investigational Component (YBOCS-IC) is composed of six items evaluating insight into obsessions and compulsions, avoidance, degree of indecisiveness, overvalued sense of responsibility, pervasive slowness and pathological doubting. The third section, is defined general (Y-BOCS-G) and evaluates, by three items, the severity of symptoms. The Y-BOCS is administered by a semi-structured interview whose main goal is to compute the scores of the Y-BOCS-CC (items 1 - 10). The remaining items are not considered in the overall score, given the lacking of systematic studies on their psychometric properties [16]. The overall YBOCS score ranges from 0 to $40 \mathrm{pts}$, to which correspond five levels of severity correspond, i.e., sub-threshold (0 - 7 pts), mild ( 8 - 15 pts), moderate (16 - 23 pts), severe (24 - 31) and extremely severe (32 - $40 \mathrm{pts}$ ). In the four subjects under investigation the levels were the following: moderate, one patient, severe, one patient, and extremely severe, two patients.

The CAT strategies category grid. Communicative interaction on video-taped material has been classified by means of the Initiative-Response Analysis grid [18]. This tool implies an analysis of interaction at its micro-level, which evaluates dynamics subtending relationships between partners across conversational turns. The turn may be intended as the coding unit in its simultaneous dimension of context-determined and context-determining.

With regard to its properties of response or retroactive part, the turn is context determined, given its retroactive part linked with the antecedent turn of the interlocutor. This linkage gives coherence and harmony to the communicative interaction. From the other hand, the turn is also context-determining, because it comprises a proactive part, which has the role of introducing well-detailed arguments, or topics which can be further developed across following turns.

The responses are defined on the way in which they are linked with turns of the interlocutor. The local responses are linked to the antecedent turn; the focal responses expand the main topic of the antecedent turn; the adjacent responses make a linkage between close (but not immediately antecedent) topics; non-local responses refer to arguments taken into account several turns before; finally, self-linking responses are the unique which are linked to the turn(s) always conveyed by the speaker but not by the interlocutor [18].

The initiatives, i.e. the turns contributing to define the arguments of conversation, are classified into three categories: directing, controlling and inhibiting. The first category includes initiatives adopted by the speaker to force the interlocutor in responding to given conditions; for example, formulating questions asking answers. This particular style is well represented by the questioning by police [19]. The second category refers to acts aiming to evaluate, correct or discredit contributions of the partner. The inhibiting initiatives include all attempts by the interlocutor against the partner to leave him apart from the opportunity to participate to the conversation.

A particular category of code has been assigned to each turn according to the I-R system, which includes 18 
different categories, plus one indicating non-classifiable turns [16].

\section{Measures}

Given the categories of the I-R system, several coefficients have been calculated to evaluate interaction strategies. Furthermore, the theoretical analogies between the coding system and accommodation strategies allowed us to use I-R codes to calculate coefficients of convergence, divergence and maintenance.

The coefficients have been expressed as percentage of frequency, with a range between 0 and 100 . They represent the percentage ratio between the sum of frequencies of codes and the total number of turns for each single speaker. The coefficients of the I-R system, assumed as variables in the present study, are reported as follows:

Coefficient Balance (B): percentage of expanded responses for each single speaker [sum of codes $<\wedge$ divided by the number of codes $\times 100]$. This coefficient indicates the tendency of the speaker to link his turn to antecedent turn of the interlocutor (local responses).

Coefficient Solicitation (S): it is given by the number of turns characterized by strong initiatives [sum of codes: $<>>; \cdots>;=>;<=>$; : $>$ divided by the number of codes $\times 100]$. This coefficient reflects the speaker's attempts in evoking responses from the interlocutor on topics proposed by the speaker himself. The coefficient includes, consequently, the directing initiatives.

Coefficient Fragmentation (F): it is the number of turns not related or non-locally related to turns of the interlocutor [sum of codes: $>; \wedge ; \cdots>; \cdots \wedge ; \cdots<;(>$ divided by the number of turns $\times 100]$. This coefficient gives the measure of sudden thematic changes acted by the speaker, which include local incoherence or fragmentation of the conversation (remote or non-local responses).

Coefficient Obliqueness $(\mathrm{O})$ : it is given by the number of turns in which the speaker avoids the linkage with the main topic of the adjacent turn of the interlocutor, not exhibiting a focal relation or linking only to his own conversational turns [sum of codes: $=>;=\wedge ;<=>;<$ $=\wedge ;: \wedge ;:>$ divided by the number of turns $\times 100]$. The coefficient $\mathrm{O}$ includes responses defined as "self-linking", i.e., responses linked to turn(s) of the speaker, not taking into account contributions from the partner.

All the coefficients directly reflect accommodation strategies, in the sense of adaptation to conversational contents. Consequently, the convergence may be assumed to be marked by the times the speaker is adherent to interaction by means of local responses linked to the antecedent turn, giving harmony to the conversation. According to this definition, the convergence may be operatively expressed by the coefficient B (balance) inside the I-R system. The divergence indicates the degree in which the speaker is non-adherent to conversational contributes of the partner. Furthermore, the divergence may be intended as differentiation by means of non-focal or non-local responses. In this sense, the divergence may be expressed by the two coefficients $\mathrm{F}$ and $\mathrm{O}$. The coefficient $\mathrm{O}$, in particular, represents the strategy that theory of CAT indicates as a form of divergence, that is, maintenance.

Bearing in mind that coefficient $\mathrm{O}$ incorporates selflinking responses; we would assume it is a reliable index of maintenance, because in self-linking responses the speaker neglects communicative contribution from the partner to "maintain" his own arguments/turns.

A sample of coded interview is reported as follows (geographical coordinates have been changed).

$1 \mathrm{I}$ : OK, let's talk about something else: the law about forbidden smoking, do you know it $?<$ ).

$2 \mathrm{C}$ : yes I do, but the law has been repealed, or not, it has been prolonged for six months more $<\wedge$ coefficient B.

3 I: now not interruption $\mathrm{X}$.

$4 \mathrm{C}$ : and by March ahead? $=\wedge$ coefficient $\mathrm{O}$.

5 I: by January, January-February, is in force the 2000 euros fine for both holders of public places and interruption $<>$ coefficient $\mathrm{S}$.

$6 \mathrm{C}$ : smokers $<\wedge$ coefficient $\mathrm{B}$.

$7 \mathrm{I}$ : do you agree? $=\wedge$ coefficient $\mathrm{O}$.

$8 \mathrm{C}:$ no $<$.

9 I: why? $=\wedge$ coefficient $O$.

$10 \mathrm{C}$ : because I am a smoker, so I do not agree $<$.

$11 \mathrm{I}$ : I see. Anyway, have you never smoked in places in which smoking was forbidden, and what did you do in this case? $<\wedge$ coefficient $\mathrm{B}$.

$12 \mathrm{C}$ : In some places yes, I have; in some others there were rooms where smoking was permitted -.

$13 \mathrm{I}$ : has it never occurred to you interruption X.

$14 \mathrm{C}$ : recently it occurs to hear something for example: please, get out of this place with your cigarette $=\wedge$ coefficient $\mathrm{O}$.

15 I: don't you believe this request in respecting the law is a way to protect peoples' health? Don't you consider it is dangerous for health? $<\wedge$ coefficient $B$.

$16 \mathrm{C}$ : they are so dangerous chemical toxic, factories, cars... -

17 I: so changing our argument, we could talk about the pollution of this city. You have just alluded to it. A way to reduce emissions by cars could be to increase moving by public transport $<\wedge$ coefficient $B$.

$18 \mathrm{C}$ : that is an economic factor, I don't know if somebody can hold an own car or not, this is a money business : $\wedge$ coefficient $\mathrm{O}$.

19 I: how do you move usually? $\wedge$.

$20 \mathrm{C}$ : only recently I have been traveled for small distances, I lived in Swiss for many years, last year I moved to Caserta to stay with my relatives ... I am not a Ber- 
lusconi's son ... : > coefficient $\mathrm{O}$.

$21 \mathrm{I}$ : so people without money must use public transport, whereas rich persons could use the car $=\wedge$ coefficient $\mathrm{O}$.

$22 \mathrm{C}$ : however, also people with money want to save something : $\wedge$ coefficient $\mathrm{O}$.

$23 \mathrm{I}: \mathrm{OK}$, are you satisfied by public transport? $\wedge$.

$24 \mathrm{C}$ : well, some time before there were small buses which reached all places around Caserta, but the service stopped because the buses were always vandalized :> coefficient $\mathrm{O}$.

25 I: I see -.

$26 \mathrm{C}$ : it is a good stuff to get the fine on forbidden smoking, although I do not agree to complete prohibit smoking, I do not accept this, because I smoke, and when I eat I want to smoke, but it is a good thing to get a fine up to 1000 euros $<=>$ coefficient $\mathrm{O}$ (self-linking).

As shown in the sample of the interview, turns 2, 6, 11, 15 and 17 report index codes to calculate the coefficient $\mathrm{B}$, i.e., the convergence. These turns represent both response and initiative. The speaker links to the antecedent turn of the partner, so contributing to the harmony of the ongoing communicative act. The turns 4, 7, 9, 14, 18, 20, $21,22,24$ and 26 are coded by indexes to calculate coefficient $\mathrm{O}$. These turns represent the tendency to re-link to own adjacent turns by the speaker, or to link to arguments close within the conversation. In the last case, it is evident that the speaker does not take into account the partner's contribution, because the partner is often interrupted, or he is only marginally involved by means of so-called non-focal linking. As a matter of fact, the turn 26 is coded as self-linking: the speaker comes back on a topic to which he had always answered by turns $1-14$.

\subsection{Reliability of I-R System}

The inter-rater reliability on coding from two independent raters has been calculated by means of the Cohen $\mathrm{K}$ (Cohen, 1960) [20,21]. Computation has been supported by the statistical package ComKappa [22] running on PC. The Cohen $\mathrm{K}$ value, computed on three out of eight interviews was 71 .

\subsection{Data Analysis}

Comparisons between measures of strategies of the two groups have been performed by non-parametric tests suitable for analyzing small samples. The analyses are summarized as follows: Mann-Whitney U standardized test $(Z)$ for independent samples (OCD group vs NC) with significance level (two-tail) at $\mathrm{p}<0.05$; Wilcoxon signed rank test for dependent samples (interviewer-interviewed), with significance level (one-tail) at $\mathrm{p}<0.05$; Pearson $r$ coefficient, with significance level (one-tail) at $\mathrm{p}<0.05$, to evaluate correlation between the clinical variables of the Y-BOCS and measures of interactive strategies. Computation has been supported by the statistical package SPSS 14, running on PC.

\section{Results}

\subsection{Descriptive Statistics of the OCD Sample}

Table 1 reports values of both whole OCD group and single participant on each evaluated variable. The strategy mainly used was the Solicitation toward the interlocutor $(38 \%)$. However, the percentage of Obliqueness, which represents the strategy of Maintenance, is also high (20\%). Then follow, with similar percentages (14\%), Fragmentation and Balance, which represent Divergence and Convergence, respectively.

\subsection{Analysis for Independent Samples}

Among all comparisons between OCD and NC on measures of strategies (given by coefficients B, S, F and O), a significant group effect was evident only on the Obliqueness $(Z=-2.30, p=0.021)$. Patients with OCD showed non-focal links and self-linking responses, on average in $20 \%$ of the cases $\left(\bar{x}_{O C D}=19.8, \mathrm{sd}=3.6\right)$. This percentage was significantly higher than that of NC $(7 \%$; $\bar{x}_{\text {CONTR }}=7.4, \mathrm{sd}=3.8$ ), see Figure 1 .

Table 1. Presence of each I-R index (balance, solicitation, fragmentation, and obliqueness) for each participant OCD and for the whole group (with standard deviation).

\begin{tabular}{ccccc}
\hline$\#$ & Balance & Solicitation & Fragmentation & Obliqueness \\
\hline 1 & 13.8 & 27.6 & 13.8 & 24.6 \\
2 & 8.3 & 26.6 & 5 & 16.6 \\
3 & 22.4 & 39.6 & 12 & 20.6 \\
4 & 13 & 58.6 & 26 & 17.3 \\
TOT. & $14.4(5.86)$ & $38.1(14.89)$ & $14.2(8.76)$ & $19.8(3.63)$ \\
\hline
\end{tabular}

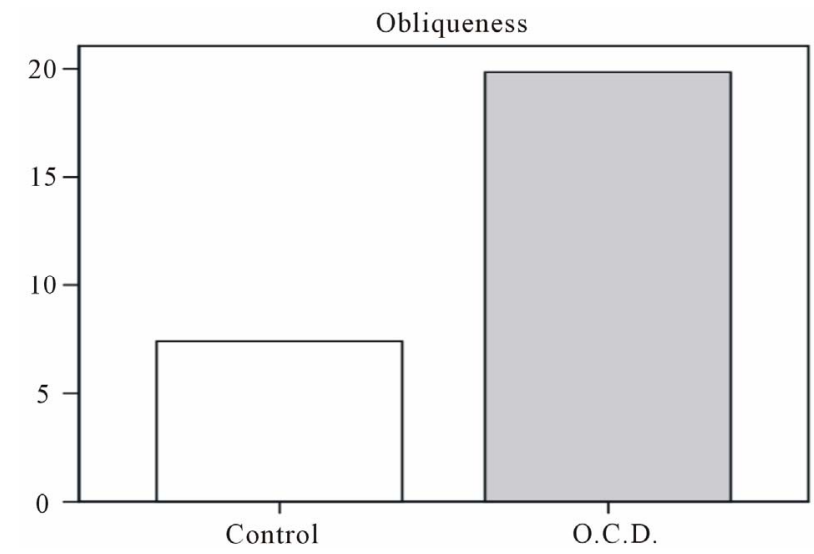

Figure 1. Mean difference in obliqueness (maintenance) between OCD and control sample. 


\subsection{Analysis for Dependent Samples}

None of interviewer-interviewed comparison within NC group approached significance. This result indicates that in $\mathrm{NC}$ the adopted interactive strategies are quite similar. Conversely, within the OCD group, the Obliqueness coefficient shows a significant trend $(\mathrm{z}=-1.82, \mathrm{p}=0.06)$, given by the higher value of this coefficient as compared to that of the interviewer $\left(13 \% ; \bar{x}_{\text {INTERVIEWER }}=13.3, \mathrm{sd}=\right.$ $6.1)$.

\subsection{Correlation between I-R Indexes and Y-BOCS Scores}

Table 2 reports correlations between coefficients of interactive strategies and scores (overall and by subscales) of the Y-BOCS in the OCD group. The only $r$ value which approached significance resulted from correlation between Compulsion subscale and the Obliqueness index, that is, interactive strategy of Maintenance. Although not strictly significant, almost all correlation showed a medium or high load. For instance, all $r$ values between Obsession subscale and I-R coefficients are positive and higher than 0.50 . This led us to believe, at least at clinical and descriptive level, that increasing obsessiveness carries with the use of strategies of Convergence, Divergence and Maintenance, with a generalized tendency to use during the interview all possible interactive strategies.

Conversely, the Compulsion subscale gives more defined results: it is associated with the Maintenance by the only significant correlation observed. Furthermore, the Compulsion is quite independent from the Solicitation index (i.e.: Convergence) and is weakly related with indexes of Balance and Fragmentation. This led us to hypothesize that tendency to Compulsion, showed by people with OCD, is exclusively related to the strategy of Maintenance.

\section{Discussion and Conclusions}

In the present study, we have investigated the characteristics of interactive strategies of Convergence, Divergence and Maintenance in subjects suffering from OCD. The results showed that these patients over-relied, in communicating, on oblique strategies of Maintenance. This issue is consistent with the hypothesis of a "rigidity" of the communicative style in patients with OCD, which led these subjects to link communication to their own conversational turns, often neglecting the communicative contributions of the partner.

This tendency to self-linking communication, neglecting contributions by the others, may be the consequence of a brooding over of thought, which forces the obsessive speaker in persisting on his own turns, or going back to continuously recheck his positions, in order to satisfy his need of being sure. This need is predominant in subjects, like OCD, which are continuously worried by doubts.

The two groups under investigation showed different results also when interviewer-interviewed comparison has been considered. In fact, NC did not show any difference on each of the evaluated indexes, thus indicating that communication of control subjects has been characterized by reciprocal interactive adaptation between partners, who both concurred to create a symmetric accommodation. In the OCD group, on the contrary, there was an over-reliance on the obliqueness index (i.e. Maintenance) regarding the interviewer. This has been supported by results of both between and within group(s) comparisons, which showed that the discriminant factor of the OCD group was the use of the interactive strategy of Maintenance.

Further issues could come from correlation analyses, which could indicate the obliqueness as specific of compulsive dimension, in the sense that the severity of compulsive symptoms carries with an increasing use of Maintenance strategies. This association could be due to the influence given by compulsions on attention functions, in particular as if concerns task performing, which includes planning and monitoring of the communicative acts.

The compulsions are repetitive motor (e.g., handwashing, rearranging, checking) but also mental behaviors (e.g., praying, counting, repeating or reciting sentences), which are recognized by the individuals as excessive or senseless, but are nonetheless necessary because of their effect in alleviating anxiety and/or neutralizing obsessions [23]. In this sense, compulsions assume the value of an exit strategy. Consistent with this view, a possible interpretation of our results points to the use of self-referential strategies as the communicative effect of thought processes, that is, a forced focusing on own topics of thought in order to reduce anxiety given by obsessions.

Table 2. Correlations of the I-R indexes with the total score of the Y-BOCS and with the two subscales of obsession and compulsion.

\begin{tabular}{ccccc}
\hline CAT Strategies & I-R Indexes & Obsession (Subtotal) & Compulsion (Subtotal) & Y-BOCS (Total score) \\
\hline Convergence & B Balance & $0.69(\mathrm{p}=0.16)$ & $0.33(\mathrm{p}=0.33)$ & $0.53(\mathrm{p}=0.23)$ \\
& S Solicitation & $0.51(\mathrm{p}=0.24)$ & $-0.03(\mathrm{p}=0.48)$ & $0.24(\mathrm{p}=0.37)$ \\
Divergence & F Fragmentation & $0.70(\mathrm{p}=0.14)$ & $0.36(\mathrm{p}=0.32)$ & $0.56(\mathrm{p}=0.22)$ \\
Maintenance & O Obliqueness & $0.58(\mathrm{p}=0.20)$ & $0.91(\mathrm{p}=0.045)$ & $0.79(\mathrm{p}=0.10)$ \\
\hline
\end{tabular}


As concluding remark, we would stress that this study may be considered as a contribution towards an integrating of clinical and communicative social psychology and a support to a "personologic" hypothesis. According to this hypothesis, intra-individual factors reflected in the interactive communicative style. As a matter of fact, results highlight the peculiarity of accommodation strategies by subjects affected by OCD. Further studies will address the role of specific personality traits in different communicative settings (e.g., clinical settings, informal context, group interaction etc.), possibly involving wider sample of subjects in order to give stronger support to theoretical framework. One of main limits of this research, indeed, is the small sample we interviewed, because of the difficulty of recruitment. From the other hand, the communicative strategies could become a further indicator of clinical diagnosis, but also interesting for the assessment of therapeutic relationship. Hence, systematic and quantitative studies on accommodation strategies could be useful, in a clinical perspective, to verify efficacy and efficiency of therapeutic programs aimed to reach higher levels of "communicative synchrony" [24].

\section{REFERENCES}

[1] C. Lee, H. Giles and R. N. Saint Clair, "The Social and Psychological Context of Language," LEA, Hillsdale, 1980.

[2] P. Robinson and H. Giles, "The New Handbook of Language and Social Psychology," John Wiley, New York, 2001.

[3] G. Bateson, "Step to an Ecology of Mind Chandler," University of Chicago Press, New York, 1972.

[4] R. Y. Bourhis, "Language in Ethnic Interaction: A Social Psychological Approach,” Pergamon, Oxford, 1979.

[5] A. Gnisci, "Sequential Strategies of Accommodation: A New Method in Courtroom," British Journal of Social Psychology, Vol. 44, No. 4, 2005, pp. 621-643. doi:10.1348/014466604X16363

[6] A. Gnisci and R. Bakeman, "Sequential Accommodation of Turn Taking and Turn Length: A Study of Courtroom Interaction," Journal of Language and Social Psychology, Vol. 9, No. 26, 2007, pp. 234-259. doi:10.1177/0261927X06303474

[7] J. M. Jan, "Code-Switching for Power Wielding: Inter Gender Discourse at the Workplace," Multilingua, Vol. 22, No. 1, 2003, pp. 41-57. doi:10.1515/mult.2003.003

[8] R. M. McCann and H. Giles, "Communication with People of Different Age in the Workplace: Thai and American Data," Human Communication Research, Vol. 32, No. 1, 2006, pp. 74-108. doi:10.1111/j.1468-2958.2006.00004.x
[9] A. R. Scott and H. Giles, "Intergroup Relations: Its Linguistic and Communicative Parameters," Group Processes \& Intergroup Relations, Vol. 8, No. 3, 2005, pp. 211-214. doi: $10.1177 / 1368430205053938$

[10] H. Ota, H. Giles and C. Gallois, "Perceptions of Younger, Middle-Aged and Older Adults in Australia and Japan: Stereotypes and Age Group Vitality," Journal of Intercultural Studies, Vol. 23, No. 3, 2002, pp. 253-266.

[11] M. K. David, "Preface," Multilingua, Vol. 22, No. 1-4, 2003.

[12] H. Tajfel, C. Flement, M. Billig and R. P. Bundy, "Social Categorization and Intergroup Behaviour," European Journal of Social Psychology, Vol. 1, No. 2, 1971, pp. 149178. doi:10.1002/ejsp.2420010202

[13] F. Mancini, "Un Modello Cognitivo del Disturbo OssessivoCompulsivo," Psicoterapia, Vol. 22-23, 2001, pp. 43-60.

[14] F. Mancini, F. D'Olimpio and S. D'Ercole, "Responsability, Attitude, Obsession e Compulsion: Further Support in a Non Clinical Sample," Clinical Psychology \& Psychotherapy, Vol. 8, No. 4, 2001, pp. 274-281. doi: $10.1002 / \mathrm{cpp} .292$

[15] A. Pedon and A. Gnisci, "Metodologia della Ricerca Psicologica,” Il Mulino, Bologna, 2004.

[16] W. K. Goodman, L. H. Price, S. A. Rasmussen, et al., "The Yale-Brown Obsessive Compulsive Scale: I. Development Use and Reliability," Archives of General Psychiatry, Vol. 46, No. 11, 1989, pp. 1006-1011. doi:10.1001/archpsyc.1989.01810110048007

[17] P. I. Marconi and F. De Palma, "La Misura Delle Ossessioni e gli Strumenti di Valutazione," In: P. Pancheri, Ed, Ossessioni, Compulsioni e Continuum Ossessivo, Il Pensiero Scientifico Editore, Roma, 1992, pp. 4175-4180.

[18] P. Linell, L. Gustavsson and P. Juvonen, "Interactional Dominance in Dyadic Communication: A Presentation of Iniziative-Response Analysis," Linguistic, Vol. 26, 1988, pp. 415-442.

[19] P. Linell, "The Power of Dialogue Dynamics," In: H. Giles and N. Coupland, Eds., Contexts of Accommodation, University Press, Cambridge, 1990.

[20] A. Gnisci and R. Bakeman, "L'osservazione e l'Analisi Sequenziale dell'Interazione,” L.E.D., Milano, 2000.

[21] R. Bakeman and A. Gnisci, "Sequential Observational Methods," In: M. Eid and E. Dieneer, Eds., Handbook of Multimethod Measurement in Psychology, APA, Washington DC, 2005, pp. 127-140.

[22] B. F. Robinson and R. Bakeman, "ComKappa: A Windows 95 Program for Calculating Kappa and Related Statistics," Behaviour Research Methods, Instruments e Computers, Vol. 30, 1998, pp. 731-733.

[23] American Psychiatric Association (APA), "Diagnostic and Statistical Manual of Mental Disorders (DSM-IV-TR)," APA, Washington DC, 2000.

[24] L. Anolli, "Psicologia della Comunicazione," Il Mulino, Bologna, 2002. 